\title{
Abdominal Scar Endometriosis: Report of Three Cases with Cytohistocorroboration
}

\author{
Ritu Arora*, Ila tyagi, Namrata Nargotra and Garima Singh and Abhijit Das \\ Department of Pathology, NDMC Medical college \& Hindu Rao Hospital, New Delhi, India
}

\begin{abstract}
Endometriosis of the abdominal wall scar is rare, reported incidence being $1.08 \%$ for mid-trimester abortion and $0.03-0.4 \%$ following caesarean sections. Incisional endometriosis is a relatively rare entity and is often misdiagnosed as stitch granuloma, abscess, incisional hernia, lipoma, hematoma, desmoid tumor, sarcoma, lymphoma, or primary and metastatic cancer.

We report three cases of abdominal scar endometriosis; two cases occurring in the surgical scar of previous caesarean incision and one case occurring after second trimester hysterotomy.

The present study describes the physical signs and symptoms and cytological features of abdominal scar endometriosis. A high index of suspicion is needed for early diagnosis of this entity in a woman presenting with post operative abdominal lump.
\end{abstract}

\section{Keywords: Endometriosis, Surgical Scar, Abdominal Wall}

\section{Introduction}

Endometriosis is defined as the presence and proliferation of the endometrium outside the uterine cavity. The most commonest site is pelvis. ${ }^{[1]}$ The sites of extra pelvic endometriosis are bowel, appendix, omentum, kidney, lymph nodes, lungs, pleura, umbilicus, hernial sacs, and abdominal wall. ${ }^{[1,2]}$

Endometriosis in an abdominal scar is rare, reported incidence being $1.08 \%$ for mid-trimester abortion and $0.03-0.4 \%$ following caesarean. ${ }^{[3,4]}$. This entity is often misdiagnosed as stitch granuloma, abscess, incisional hernia, lipoma, hematoma, desmoid tumor, sarcoma, lymphoma, or primary and metastatic cancer. ${ }^{[4]}$ Due to non specific symptoms and variable presentation, the diagnosis is often delayed or missed. The present study describes 3 cases of scar endometriosis with the cytological features, physical signs and symptoms that may lead to earlier diagnosis and treatment.

\section{Case Report(S)}

Case 1: A 30-year-old female (G4P3) presented with a nodule over anterior abdominal wall scar, 8 month after second trimester hysterotomy. It was associated with increase in size and pain during menstrual cycle. Examination revealed a well-defined, $3 \times 3 \mathrm{~cm}$ tender and hyperpigmented nodule over the anterior abdominal hysterotomy scar (Figure 1). USG revealed a heteroechoic area in anterior abdominal wall. Clinical diagnosis of endometriosis was suggested.
Case 2: A 25 year old female presented with a 2 month history of persistent and continuous pain on anterior abdominal wall and had history of caesarian section 3 years back. Physical examination revealed a well-healed caesarian scar, with a non-mobile, nodular swelling at its lateral border. Clinical diagnosis of hypertrophied scar was suggested.

Case no 3: A 28 yr old female presented with a nodule associated with ulceration over anterior abdominal wall. Patient had undergone caesarean section 6 months back. Clinical diagnosis of abscess was suggested.

In all three cases, FNAC was done. Cytological smears showed monolayered sheets of cohesive epithelial cells, stromal fragments of spindle cells with ovoid or elongated nuclei along with naked stromal nuclei and hemosiderin laden macrophages (Figure 2). Epithelial cells have scant cytoplasm, uniform round to oval nuclei with bland chromatin and inconspicuous nucleoli. In our sudy, one case shows cytoplasmic microvacuolistion consistent with secretory phase of menstrual cycle (figure 3 ). Hemosiderin laden macrophages were present in two cases. (Figure 4). Based on these features, FNAC diagnosis of endometriosis was suggested.

Nodules were excised and submitted for histopathology. Grossly, case one measured $3 \times 3 \times 1.0 \mathrm{~cm}$, other two cases measured $2.0 \times 1.5 \times 1.5 \mathrm{~cm}$ and $2.5 \times 2 \times 1.5 \mathrm{~cm}$ respectively. Cut section shows fibrofatty tissue with central fibrous grey-white area containing minute cystic spaces 
(figure 5) Microscopy revealed irregularly dilated endometrial glands surrounded by endometrial stroma with scattered

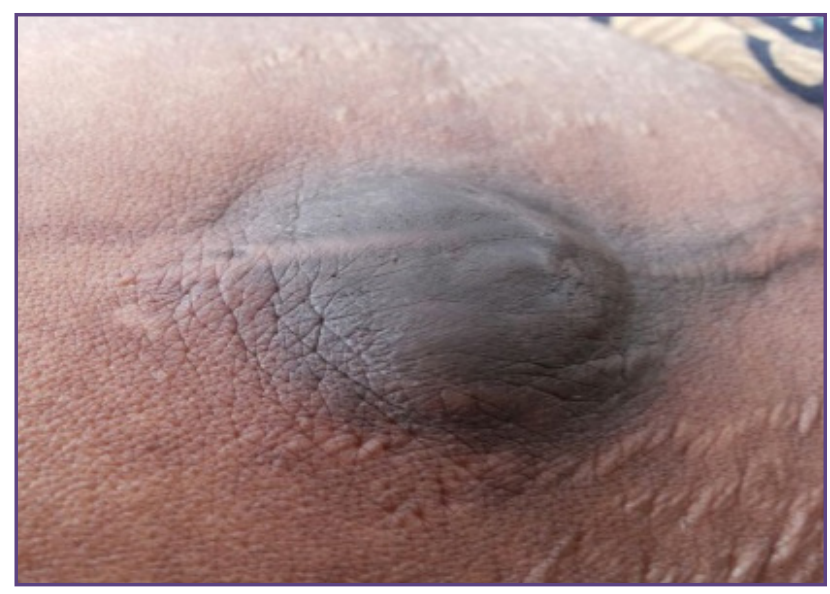

Fig. 1: Nodule on surgical scar over abdominal wall.

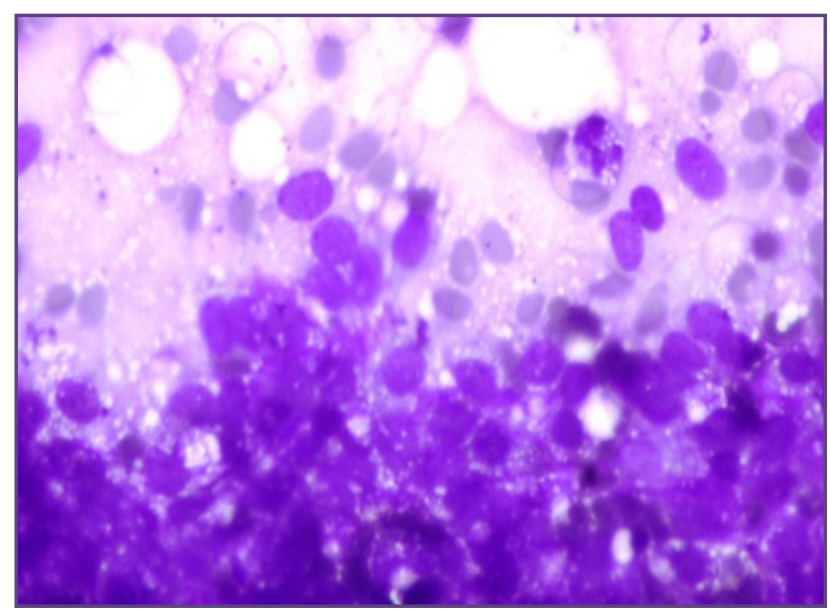

Fig. 3: Cytological smears showing endometrial cells with vacoulisation (MGG, x400).

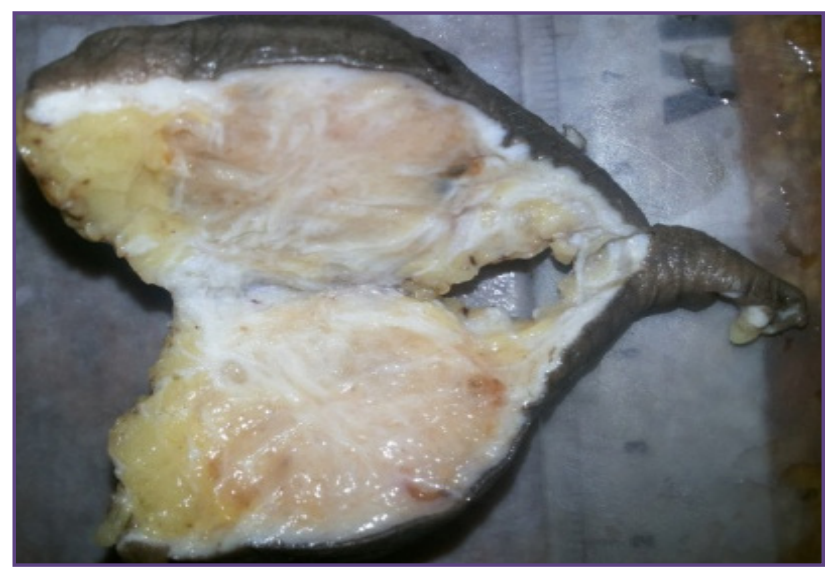

Fig. 5: Photomicrograph showing excised specimen of the abdominal wall swelling. $\mathrm{C} / \mathrm{S}$ shows gray-white areas of endometriosis. hemosiderin laden macrophage within the fibrocollagenous tissue confirming endometriosis. [Figure 6]

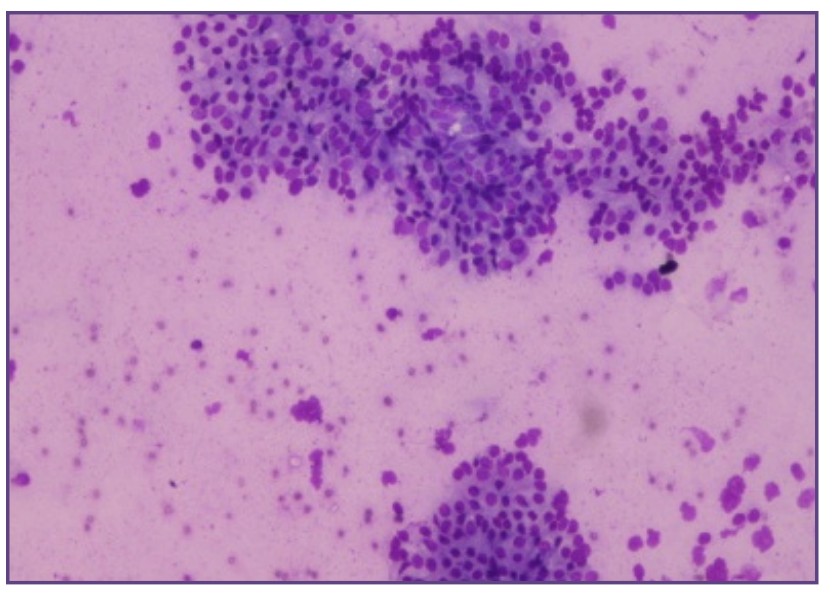

Fig. 2: FNA smear showing monolayered sheets of endometrial and stromal cells.(MGG, x100)

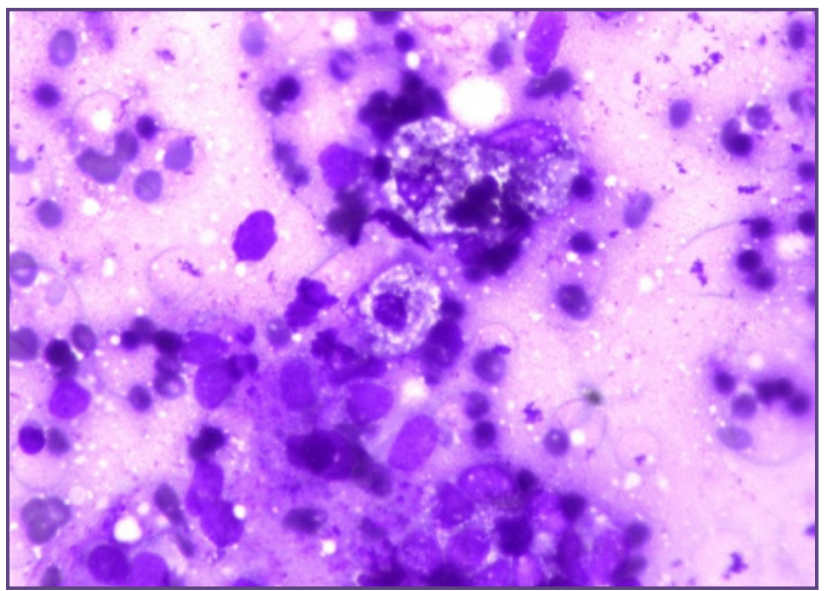

Fig. 4: Photomicrograph shows endometrial cells and hemosiderin laden macrophages (arrow) MGG,X400).

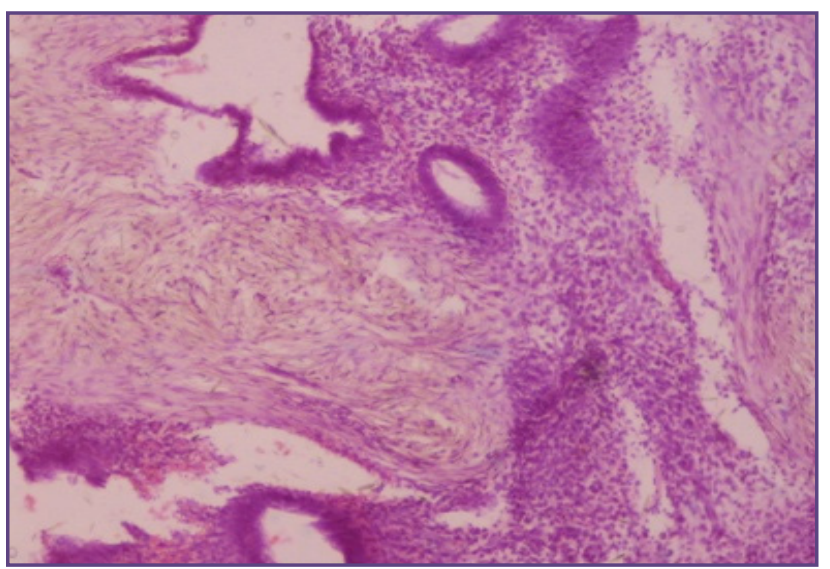

Fig. 6: FPhotomicrograph showing cystically dilated endometrial glands surrounded by endometrial glands in a fibrous stroma. ( $H$ and $E, \times 100)$. 


\section{Discussion}

Incisional endometriosis is relatively rare entity and usually occurs following obstetric or gynaecologic surgeries. It can occurs at the skin, subcutaneous tissue, rectus muscle/ sheath, intraperitoneally or in the uterine myometrium (within uterine scar).

The diagnosis of scar endometriosis is usually challenging. Patients usually complain of constant abdominal pain difficult to localise, or a tender lump in incisional area. However a mass overlying a scar associated with symptoms of cyclic pain related to menses is nearly pathognomonic of endometriosis, these classical symptoms are present only in $20 \%$ of the patients. ${ }^{[6]}$ In present study only one patient presented with cyclical changes of pain and increase in size of nodule, suggestive of classical endometriosis. In one of our case overlying discolouration of mass was seen and in one case mass was associated with ulceration leading to clinical diagnosis of abscess.

The interval between onset of symptoms and patient's surgery may varies from 3 months to 10 years. ${ }^{[7]}$ In this study, the interval between surgery and onset of endometriosis symptoms was between 6 months 3 years. ${ }^{[8]}$

In the majority of the cases the sonographic appearance of abdominal wall endometriosis are nonspecific describing the lesion as cystic, multicystic, mixed, or solid . So needle biopsy is the diagnostic tool in confirming the diagnosis. [9] In patients with incisional endometriosis, 25\% have concomitant pelvic endometriosis. ${ }^{[10]}$ In our study there was no sonological or medical evidence of pelvic endometriosis in either case.

Cytologically, smears show variable cellularity. The presence of any two of three cytological components (endometrial glands, stromal cells and hemosiderin laden macrophages) can be used for cytological diagnosis of endometriosis. ${ }^{[11]}$ Cytological features are related to hormonal changes due to the functional nature of endometriosis. In proliferative phase, epithelial cells form cohesive sheets of uniform small cells with scant cytoplasm, round to ovoid nuclei with bland chromatin and occasional non atypical mitosis. During secretory phase, cytoplasmic microvacuolations and nuclear atypia occurs in glandular cells. Stromal cells may show decidual or myxoid change causing diagnostic difficulties. ${ }^{[11]}$ In very rare instances endometriosis may undergo malignant change. Carcinoma associated with endometriosis are most often endometrioid $(70 \%)$ and clear cell $(14 \%) .{ }^{[12]}$ In our study, hemosiderin laden macrophages were present in two cases. One case shows cytoplasmic microvacuolistion consistent with secretory phase of menstrual cycle.
Clinically this entity is often misdiagnosed as suture granuloma, abscess, fat necrosis, hematoma, fibromatosis, sarcoma, lymphoma, or primary and metastatic carcinoma. ${ }^{[4]}$ FNAC is a rapid, cheap and effective tool in distinguishing these lesions from endometriosis.

Abdominal fibromatosis can be confused with endometriosis in pregnant females especially in areas of caesarean section scar. A presurgical FNA can help to avoid misdiagnosis. Cytosmears shows variable cellularity with spindle shaped fibroblasts and interspersed collagenenous stroma. Epithelial component of endometriosis is lacking.

Cytosmears from primary or metastatic adenocarcinoma are hypercellular with presence of frankly neoplastic cells while there is bland cytonuclear morphology of glandular component in endometriosis. ${ }^{[12]}$ Smears in soft tissue sarcoma are cellular with atypical bizarre spindle cells, atypical mitosis and multinucleated giant cells. ${ }^{[13]}$

Stitch granuloma is a benign granulomatous condition occurring in response to non absorbable suture. Cytologically, it is characterized by mixed inflammatory infiltrate, foreign body giant cells with presence of necrotic debris, fibrinous material and fragments of suture material in the background. Fat necrosis shows fragments of adipose tissue, foamy macrophages, inflammatory and multinucleate giant cells, and no epithelial cells. Thus history, clincoradiological correlation and FNA can help to avoid mistakes in approach of abdominal wall tumors.

Management includes both surgical excision and hormonal suppression. ${ }^{[14,15]}$ It is believed that hormonal suppression is only partially effective and surgical excision of the scar is the definitive treatment. ${ }^{[15,16]}$

\section{Conclusion}

Abdominal incisional endometriosis can cause diagnostic difficulty due to its relative rarity and variable presentation. Hence, a high index of suspicion is recommended when a woman presents with post operative abdominal lump. FNAC is a valuable diagnostic tool for endometriosis and helps in excluding other lesions like metastatic deposit, desmoid tumor, lipomas, sarcomas, myxoma, fat necrosis, hematoma or abscess.

\section{Acknowledgements}

We would like to thank our consultants of Department of Pathology, Hindu Rao Hospital for their guidance, otherwise it would not have been possible to work on it.

\section{Reference}

1. Agarwal A, Fong YF. Cutaneous endometriosis. Singapore Med J 2008;49:704-9. 
2. Horton JD, Dezee KJ, Ahnfeldt EP, Wagner M. Abdominal wall endometriosis: a surgeon's perspective and review of 445 cases. Am J Surg 2008;196:207-12.

3. Sengul I, Sengul D, Kahyaoglu S, Kahyaoglu I. Incisional endometriosis: A report of 3 cases. Can J Surg 2009;52:444-5.

4. Park SB, Kim JK, Cho KS. Sonography of endometriosis in infrequent sites. J Clin Ultrasound 2008;36:91-7.

5. Chambers DC. Endometriosis of the abdominal surgical scar following hysterotomy. J Nat Med Assoc 1975;67:465-7.

6. Gajjar KB, Mahendru AA, Khaled MA. Caesarean scar endometriosis presenting as an acute abdomen: a case report and review of literature. Arch Gynecol Obstet 2008;277:167-9.

7. Goel P, Sood SS, R, Dalal A. Cesarean scar endometriosis - Report of two cases. Indian J Med Sci 2005; 59:495-498.

8. Medeiros FD, Cavalcante DI, Medeiros MA, Eleuterio J. Fine-Needle aspiration cytology of scar endometriosis: Study of seven cases and literature review. Diagn Cytopathol 2011;39:18-21.

9. Aydin O. Scar endometriosis. A gynaecologic pathology often presented to the general surgeon rather than the gynaecologist: Report of two cases. Langenbecks Arch Surg 2007;392:105-9.
10. Wolf Y, Haddad R, Werbin N, Skornick Y, Kaplan O. Endometriosis in abdominal scars: A diagnostic pitfall. Am Surg 1996;62:1042-4.

11. Pathan SK, Kapila K, Haji BE, Mallik MK, Al-Ansary TA, George SS, et al. Cytomorphological spectrum of scar endometriosis: A study of eight cases. Cytopathology 2005;16:94-9.

12. Catalina-Fernández I, López-Presa D, Sáenz-Santamaria J. Fine needle aspiration cytology in cutaneous and subcutaneous endometriosis. Acta Cytol 2007;51:380-4.

13. Gonzalez-Campora R. cytoarchitectural findings in the diagnosis of primary soft tissue tumors. Acta Cytol 2001;45:115-144.

14. Wolf G, Singh K. Cesarean scar endometriosis: A review. Obstet Gynecol Surv 1989;44:89-95.

15. Schoelefield HJ, Sajjad Y, Morgan PR. Cutaneous endometriosis and its association with caesarean section and gynaecological procedures. J Obstet Gynaecol 2002;22:553-4.

16. Wasfie T, Gomez E, Seon S, Zado B. Abdominal wall endometrioma after cesarean section: A preventable complication. Int Surg 2002;87:175-7.

*Corresponding author:

Dr Ritu Arora, Plot no 7, F/F, Partap Nagar, Hari Nagar, New-Delhi- 110064, India

Phone: +91 8826048344

Email: ritu_rm7@yahoo.co.in

Date of Submission : 12.08.2016

Date of Acceptance : 28.12.2016

Financial or other Competing Interests: None.
Date of Publication : 23.02.2017 